\title{
Multiscale Lattice Boltzmann modeling of two-phase flow and retention times in micro-patterned fluidic devices
}

\author{
Agnieszka Truszkowska ${ }^{\mathrm{a}, *}$, P. Alex Greaney ${ }^{\mathrm{b}}$, Goran Jovanovic ${ }^{\mathrm{a}}$ \\ ${ }^{a}$ Oregon State University, School of Chemical, Biological, and Environmental Engineering, Corvallis, OR 97331, USA \\ ${ }^{b}$ Department Mechanical Engineering, University of California - Riverside, Riverside, CA 92521, USA
}

\begin{abstract}
Recent advances for fabricating micro-featured architectures such as posts or pillars in fluidic devices provide exciting opportunities for multiphase ow management. Here we describe a novel, multiscale modeling approach for two-phase flows in microfeatured architectures developed within the Shan and Chen Lattice Boltzmann method. In our approach a fine scale is used to resolve the true microfeatured architecture, with a coarser scale used to model the gross geometry of the device. We develop the basic features of the approach and demonstrate its applicability to modeling retention times of droplets of a dispersed phase in an array of microposts - an architecture used in microfluidic reactors, bioreactors, and biomedical devises. Additionally we show that it is feasible to model the microfeatured geometry in a piecewise manner which includes extrapolating dispersed phase flow characteristics in the entire system based on simulations in smaller subdomains.
\end{abstract}

Keywords: Multiscale modeling, Lattice Boltzmann method, Two-phase flow, Microtechnology

\section{Introduction}

Microtechnology and process intensification are gaining an increasingly larger role in the engineering design of emerging technologies. A major impetus in introducing microscale-based technologies emerges from fundamental, architectural, economical, and safety/security advantages. As a technical approach a microscale-based engineering design establishes directions for potentially innovative solutions in many aspects

\footnotetext{
${ }^{*}$ Corresponding author, phone number: 1-541-908-9205

Email address: truszkoa@oregonstate.edu (Agnieszka Truszkowska)
}

(C) 2016. This manuscript version is made available under the Elsevier user license http://www.elsevier.com/open-access/userlicense/1.0/ 
of technology design (intensification of mass and heat transfer, intensification of chemical process rates, chemical product distribution and yield, process control, process safety, process scale-up) and applications (organic and inorganic chemical synthesis and processing, unit operations, environmental technologies, energy conversion technologies, renewable energy technologies). A comprehensive review of these topics can be found in Jensen (2001). For more general considerations of microreactors alone we refer to review articles such as Kolb and Hessel (2004); Kiwi-Minsker and Renken (2005); Hartman and Jensen (2009).

Multiphase flows in microscale structures provide additional complexity and opportunity for the engineering design. Novel designs of multiphase systems in chemical reaction processes (Hessel et al., 2005; Günther and Jensen, 2006), phase separations (Lam et al., 2013; Wiesegger et al., 2013), mixers (Zhao et al., 2006), and analytical devices (Huh et al., 2002), are all evidences for the proliferation of microscale-based technologies. Multiphase flows can also be an undesirable side effect occurring during device operation; a scenario that can be a consequence of normal process performance such as boiling in microscale heat exchangers. Often two-phase flow originates through introduction of the unwanted phase into the system from its surroundings, such as leaks of air bubbles entering the system through equipment gaps or with process feed (Gravesen et al., 1993; Jensen et al., 2004; Sparks et al., 2007). An unaccounted second phase may lead to systems' reduced efficiency, malfunctions and damage. Elimination of undesirable phase in microscale-based devices is often difficult, thus, a large part of research efforts are focused on proper accommodation of unwanted phases and their influence. Doku et al. (2005) provides an outline of different multiphase microreactor designs, whereas Kohnle et al. (2002); Litterst et al. (2004); Chung et al. (2008) report on microchannel based architectural solutions for improved bubble mobility.

Microscale architectures with features such as microposts or pillars partially or fully replacing classic microchannel arrays, represent a relatively unexplored and advantageous design option. Deployment of microposts in microscale-based devices creates flexible technical solutions. The introduction of microposts has the potential for addressing numerous microscale-based process requirements due to conveniently positioned internal surface areas for catalyst deposition and new opportunities for manipulation of two-phase flow. The benefits of chemical processes with solid catalysts is emphasized in Doku et al. (2005) whereas their application in microscale heat exchangers is experimentally and theoretically investigated in Krishnamurthy and Peles (2008).

Theoretical and mathematical models for multiphase flows in microscale-based structures differ substantially from macroscale systems due to the small characteristic dimensions of microscale features, and very large aspect ratios. In immiscible fluid mixtures, interface physics becomes a major contributor to fluid dynamics, and eat and mass transfer. Interfacial and viscous forces often dominate over inertia and buoyancy. In addition, the proximity of walls, the presence of architectural microfeatures, and catalysts coatings create important fluid-solid interactions. The hydrodynamic characteristics of gas-liquid flows in pillared microstructures were experimentally investigated and compared to conventional theoretical models and correlations by Krishnamurthy and Peles (2007) and De Loos et al. (2010). Results varied from established 
models and furthermore Krishnamurthy and Peles (2007) defined a new two-phase flow pattern regime, bridge flow, observed in micropillar arrays.

Design opportunities for micropillared complex architectures therefore include not only geometric considerations, but also variables such as solid affinities towards different phases, surface tension manipulation, surface modification of posts with functional chemical groups, and others. Comprehensive and efficient design requires computational tools though the benefits of numerical simulations are not confined to the design aspects only (Jensen, 2001). The importance of interfaces, geometric complexity, and commonly occurring device high aspect ratios imply high resolution and large computational efforts. Multiscale modeling provides the capacity for sufficiently accurate simulations with reasonable computational costs.

We propose a novel, multiscale modeling approach for numerical simulations of two-phase flow in complex microarchitectures developed within framework of the Lattice Boltzmann method. Due to its capabilities in representing fluid-fluid and fluid-solid interactions. The proposed approach can be extended to multiphase systems but here we present results obtained for two-phase flow only.

We focus on multiscale modeling of dispersed phase retention time. We perform simulations with fully resolved microfeatured geometry based on which we develop a force operator that when applied on the dispersed phase in an empty geometry yields sufficiently similar dispersed phase retention time. The force operator is robust and once developed becomes applicable for a range of dispersed phase sizes and operating conditions. This provides an opportunity for reducing computational effort by replacing part of the simulations in the geometrically resolved domain with computationally less intensive empty domain. Additionally, we demonstrate that it is possible to extrapolate information on the flow based on piecewise simulations instead of the entire domain. This feature is an especially important capability of the proposed approach as it could enable accurate computations of systems previously too large to simulate.

\section{Description of multiscale modeling approach}

\subsection{Scope}

The proposed multiscale modeling approach is suitable for the modeling of two-phase flow phenomena in microscale-based structures with features such as posts and pillars (Fig. 1). The repetitive design of these microarchitectures enables identification of representative regions that can provide the bases for estimates of a global behavior similar to representative volumes and unit cells in porous media.

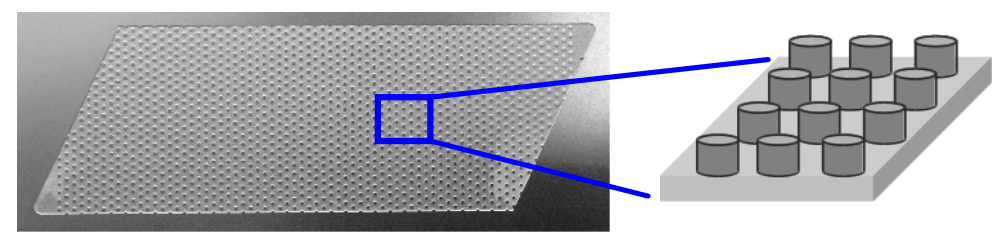

Figure 1: Microscale device with cylindrical pillars as schematically displayed. Device image courtesy of Microproducts Breakthrough Institute. 
The multiscale modeling approach we propose here has the ability to communicate critically needed information from locally highly resolved model or domain into a global, less resolved model/domain. An observed phenomenon could emerge from considerations of mass or heat transfer, chemical kinetics, characteristic process times, specific surface area, presence of energy fields/forces, interfacial forces, or any other observable occurrence which arises from structural, operating or process dynamic characteristics of the microscale-based structure.

Example of variables that can undergo proposed multiscale modeling are dispersed phase retention time, flow direction and chemical reactivity. Here we demonstrate the applicability of our approach on dispersed phase retention time which we define as the time needed for the dispersed phase to cross either the whole system in question or different subsections of the system.

\subsection{Components}

We distinguish two modeling levels that represent fine and coarse modeling scales connected through an information carrier, coupling operator.

- Fine Scale (lower level)

The fine scale or lower level is the entire microscale-based structure or its subdomain with fully resolved architecture such as posts and pillars. In general, this level requires implementation of finer computational grids to capture correct inter-fluid and fluid-solid interactions.

- Coarse Scale (upper level)

The coarse scale or upper level is the same structure without the micro-features, an "empty" or geometrically uniform domain. This level retains only gross architectural features such as walls, inlets and outlets. The computational grid on this level can be significantly coarser than in the fine scale simulations.

- Coupling operator

The coupling operator ensures transfer of information from fine to coarse scales. Here it provides sufficiently close retention time of dispersed phase on the coarse scale as on the fine scale. The coupling operator is a discrete scalar or vector field applied on the interface of the dispersed phase. In the case of retention time it is a vector field with the vector quantities referred to as forcing terms or interfacial forcing terms.

The forcing terms magnitudes may be constant throughout the system and, in which case the upper level simulations model the dispersed phase behavior as average over the entire device. The magnitudes can also have different values in distinct sections of the device such as in or around inlet or outlet regions, or in the bulk of a post array, leading to separated effective behavior in each section considered. The direction of the coupling operator depends on the influence of system architecture and flow parameters on the dispersed phase. The information passed to the upper level through the coupling operator is reduced. For example, 
in the case of retention time, the shape of the droplets of a dispersed phase is not explicitly accounted for, but its net effect is incorporated into the obtained operator. The thickness of the interface on which the forcing terms act is a variable of choice. In the Shan and Chen LBM interfaces between phases have a finite thickness of at least several computational nodes. In the simulations presented here the interface influenced was roughly half of the interfacial region from the side of the dispersed phase which corresponds to 3-4 computational nodes.

\subsection{Procedure}

The basic concept for the modeling procedure of dispersed phase flow is shown schematically in Figure 2. On the fine scale where detailed architectural features are included, flow of the dispersed phase is mostly influenced by capillary and viscous forces. On the coarse scale, the capillary forces become negligible and inertia starts dominating along with viscous forces. The coupling operator serves as a correction for the net effect of the neglected capillary forces on the coarse scale. The net effect is a change of velocity or direction of the dispersed phase when flowing through the microfeatured structure. By applying the coupling operator on the interface of the dispersed phase the influence of the microfeatures is restored to the degree of accuracy necessary for the given problem.

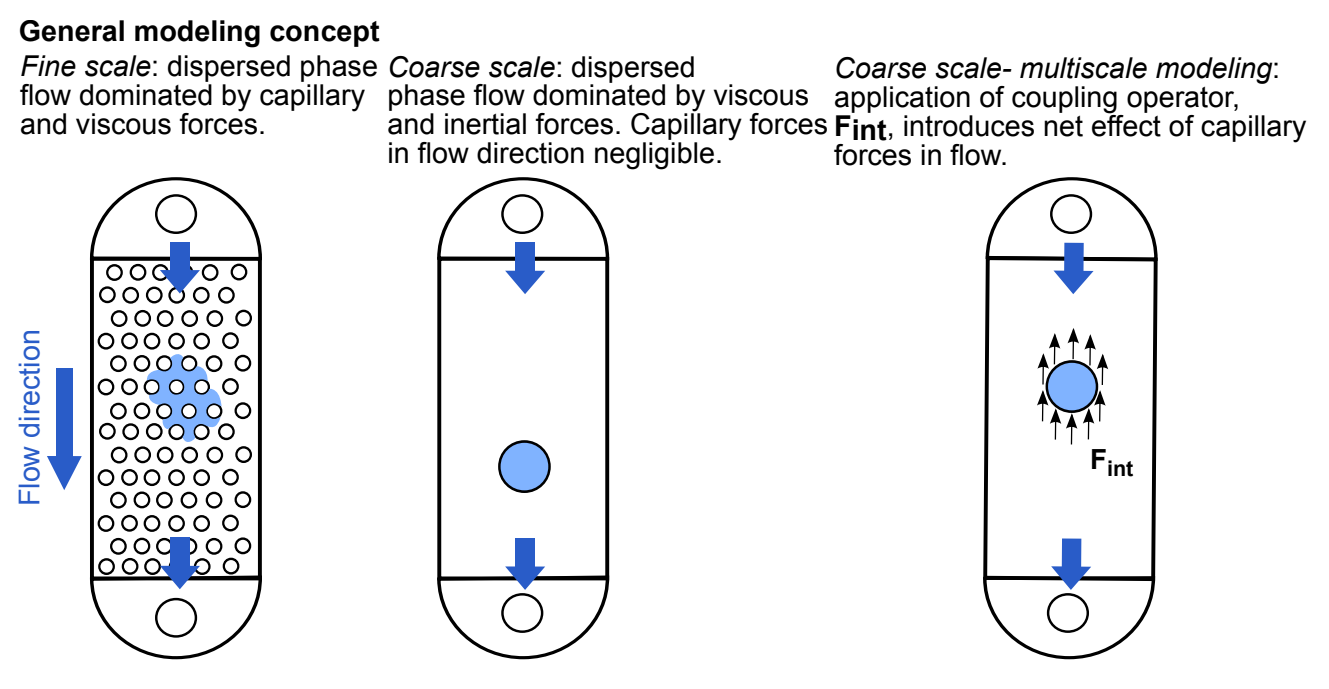

Figure 2: Proposed multiscale modeling concept for multiscale modeling of dispersed phase flow.

Development of the coupling operator consist of three steps: 1) construction, 2) implementation and 3) verification. Construction of the operator begins with two-phase flow simulations on the lower and upper levels. First the upper level is modeled without applying the coupling operator in order to determine the dispersed phase retention time relative to the lower level and the corresponding force directions. After this, the coupling operator magnitudes are determined as a fitted parameter. Through this fitting approach the coupling operator is found by an iterative back and forth communication of simulations at the upper and lower levels. The procedure is automated and can be done on the fly. The coupling operator can be computed with any desired accuracy up to a single LBM time step. In physical units, the precision of the operator 
would thus vary with the grid size and adopted scaling as described in Section 5.2. Often, as in this work, coarser operator approximations prove sufficient. Possibilities for obtaining an analytical expression for the coupling operator were explored, but while we understand the phenomenon giving rise to the friction force the coupling between the carrier flow, bubble-shape, and post array is complex making explicit derivation of this operator intractable. Meanwhile, as there exist clear physical trends in operator values, with progress of simulations one can build an operator library that correlates flow and post setups to operator values. Using such correlations would provide good initial guesses for the iterative procedure or even sufficiently accurate, ready to use, operator values.

The last step, verification, is performed separately and is referred herein as the operator robustness. By robustness we define broadness of the applicability or transferability of the developed coupling operator. It is expected that if the operator values mainly depend on the geometrical structure, the coupling operator obtained for a specific dispersed phase size and continuous fluid velocity should also be applicable for an interval of different dispersed phase sizes and continuous fluid velocities.

The verification or robustness study involves reusing the coupling operator obtained in steps 1) and 2) above in simulations with the same microarchitecture, but different operational conditions and dispersed phase sizes. This is an important step, as without a degree of robustness the proposed modeling approach does not provide any computational benefit. Hence, through this procedure we measure the range of validity of the obtained coupling operator, estimate errors and decide whether it is sufficiently accurate and robust for the given problem.

The approach is intended to model the upper level with a coarser grid than the lower level as the need for refinement of small segments in between microfeatures ceases. Utilization of coarser grid provides a first opportunity for reduction in computational effort as long as the coupling operator is sufficiently robust.

We distinguish two modeling strategies, modeling of the whole system and piecewise modeling. In the first case, fine and coarse scales keep their original dimensions throughout the modeling and operator development procedure. In contrast, in piecewise modeling instead of modeling the entire architecture on the fine scale we model only representative portions of the architecture such as inlet and outlet areas and the bulk of the post region. These regions are transformed into subdomains of smaller sizes than the original target domains in order to retrieve the coupling operator. Once the coupling operator is obtained locally within the subdomains it's values are extrapolated to cover the entire structure. This approach provides the possibility for simulating architectures currently too computationally intensive.

In this work we implement the proposed multiscale modeling approach on two simple systems with rectangular microfeatures. We obtain coupling operators and investigate their properties, alternative forms and robustness. We demonstrate the proposed approach on both an upper level with the same grid size as the lower level and an upper level with a significantly coarser grid. Finally, we present piecewise modeling results and develop criteria for subdomain construction. 


\section{Lattice Boltzmann method and proposed multiscale extension}

\subsection{Shan and Chen Lattice Boltzmann method}

The Lattice Boltzmann method is a mesoscopic modeling method based on kinetic theory of gasses in which each computational node represents a group of pseudo-fluid molecules. We utilize the Shan and Chen (SC) type LBM framework which is suitable for our multiscale extension (Shan and Chen, 1993). SC LBM has been successfully applied in modeling of various multiphase phenomena such as Laplace law or spinodal decomposition (Sukop and Thorne, 2007). In Porter et al. (2009) we find SC LBM modeling of two-phase flows through porous media with good agreement between numerical results and experimental data for air/water systems. SC LBM modeling of the multiphase flow through microchannel geometries has also been successfully validated with experimental observations by Yu et al. (2007) and Wang et al. (2011). A comprehensive overview of SC LBM as well as other methods from LBM class for modeling of multiphase flows can be found in Huang et al. (2015).

SC LBM consists of solving the density distribution function in the $a^{\text {th }}$ discrete velocity direction, $f_{a}^{\sigma}$ of each modelled species $\sigma$,

$$
\underbrace{f_{a}^{\sigma}\left(\mathbf{x}+\mathbf{e}_{a} \Delta t, t+\Delta t\right)=f_{a}^{\sigma}(\mathbf{x}, t)}_{\text {Streaming }}-\underbrace{\frac{\Delta t}{\tau_{\sigma}}\left[f_{a}^{\sigma}(\mathbf{x}, t)-f_{a}^{\sigma, e q}(\mathbf{x}, t)\right]}_{\text {Collision }}
$$

$\tau_{\sigma}$ is the relaxation time that is related to the kinematic viscosity by $\nu_{\sigma}=c_{s}^{2}(\tau-0.5 \Delta t) . c_{s}$ is the lattice speed and measures $c_{s}=c / \sqrt{3}, c=\Delta \mathbf{x} / \Delta t . \Delta \mathrm{x}$ is lattice spacing and $\Delta t$ is the time step, in this work measuring 1 lattice unit (lu) and 1 time step (ts). $f_{a}^{\sigma, e q}$ is the equilibrium density distribution function,

$$
f_{a}^{\sigma, e q}(\mathbf{x}, t)=w_{a} \rho_{\sigma}\left[1+\frac{\mathbf{e}_{a} \cdot \mathbf{u}_{\sigma}^{e q}}{c_{s}^{2}}+\frac{\left(\mathbf{e}_{a} \cdot \mathbf{u}_{\sigma}^{e q}\right)^{2}}{2 c_{s}^{4}}-\frac{\left(\mathbf{u}_{\sigma}^{e q}\right)^{2}}{2 c_{s}^{2}}\right]
$$

with $\mathbf{e}_{a}$ being discrete velocities. For the D2Q9 lattice with two spatial dimensions and nine directions these are,

$$
\left[\begin{array}{lll}
\mathbf{e}_{0}, & \mathbf{e}_{1}, & \mathbf{e}_{2}
\end{array}, \mathbf{e}_{3}, \mathbf{e}_{4}, \mathbf{e}_{5}, \mathbf{e}_{6}, \mathbf{e}_{7}, \mathbf{e}_{8}\right]=\left[\begin{array}{l}
e_{x} \\
e_{y}
\end{array}\right]=c\left[\begin{array}{ccccccccc}
0 & 1 & 0 & -1 & 0 & 1 & -1 & -1 & 1 \\
0 & 0 & 1 & 0 & -1 & 1 & 1 & -1 & -1
\end{array}\right]
$$

with $w_{a}=4 / 9(a=0), w_{a}=1 / 9(a=1,2,3,4), w_{a}=1 / 36(a=5,6,7,8) . \mathbf{u}_{\sigma}^{\mathbf{e q}}$ is the equilibrium velocity, $\mathbf{u}_{\sigma}^{e q}=\mathbf{u}^{\prime}+\frac{\tau_{\sigma}}{\rho_{\sigma}} \mathbf{F}_{\text {tot }} . \mathbf{u}^{\prime}$ is the composite velocity that combines velocities and densities of all modeled species, $\mathbf{u}^{\prime}=\frac{\sum_{\sigma} \frac{1}{\tau_{\sigma}} \sum_{a} f_{a}^{\sigma} \mathbf{e}_{a}}{\sum_{\sigma} \frac{1}{\tau_{\sigma}} \rho_{\sigma}}$.

$\mathbf{F}_{\text {tot }}$ is the total force acting on each fluid node. In a two-phase flow system it consists of forcing terms originating from attractive interactions within the same species,

$$
\mathbf{F}_{a, \sigma}(\mathbf{x}, t)=-G_{\sigma} \psi_{\sigma}(\mathbf{x}, t) \sum_{a} w_{a} \psi_{\sigma}\left(\mathbf{x}+\mathbf{e}_{a} \Delta t, t\right) \mathbf{e}_{a}
$$

repulsive interactions between different species,

$$
\mathbf{F}_{r, \sigma}(\mathbf{x}, t)=-G_{\sigma \bar{\sigma}} \rho_{\sigma}(\mathbf{x}, t) \sum_{a} w_{a} \rho_{\bar{\sigma}}\left(\mathbf{x}+\mathbf{e}_{a} \Delta t, t\right) \mathbf{e}_{a}
$$


and interactions with solids,

$$
\mathbf{F}_{s, \sigma}(\mathbf{x}, t)=-G_{s, \sigma} \rho_{\sigma}(\mathbf{x}, t) \sum_{a} w_{a} s\left(\mathbf{x}+\mathbf{e}_{a} \Delta t, t\right) \mathbf{e}_{a}
$$

$G_{\sigma}, G_{\sigma \bar{\sigma}}$ and $G_{s, \sigma}$ are interaction parameters. $G_{s, \sigma}$ depends on the wettability of the given fluid and can be calibrated or estimated to reproduce the desired material-fluid set. $\psi_{\sigma}$ (Eq.6) is an interaction potential which is a function of fluid density. $s$ (Eq.8) is an indicator function equal to 1 at the nodes occupied by solid and 0 elsewhere.

Following Buick and Greated (2000) volume, driving force, $\mathbf{F}_{\text {bulk }}$ is included between the collision step and the streaming step (Eq.1). $\mathbf{F}_{b u l k}$ forcing term is added to intermediate distribution function, $f_{a}^{\sigma^{\prime}}$ values as,

$$
f_{a}^{\sigma^{\prime \prime}}=f_{a}^{\sigma^{\prime}}+\mathbf{F}_{b u l k}=f_{a}^{\sigma^{\prime}}+\beta f_{b u l k} \mathbf{e}_{a, b u l k}=f_{a}^{\sigma^{\prime}}+\frac{1}{6} f_{b u l k} \mathbf{e}_{a, b u l k}
$$

yielding $f_{a}^{\sigma^{\prime \prime}}$ that enters the streaming step of the algorithm. $f_{b u l k}$ is the magnitude of the bulk force and vector $\mathbf{e}_{i, \text { bulk }}$ specifies force direction. $\beta=\frac{D}{Q c_{n}^{2}}$ with $D=2$ and $Q=9$ while $c_{n}^{2}=c_{s}^{2}(D+2)$.

We incorporate only repulsive interactions between different species which is a common assumption encountered in literature (Huang et al., 2007). Macroscopic densities and velocities for each component $\sigma$ are $\rho_{\sigma}=\sum_{a=0}^{8} f_{a}^{\sigma}, \quad \mathbf{u}_{\sigma}=\sum_{a=0}^{8} f_{a}^{\sigma} \mathbf{e}_{a}$ and for the entire system are $\rho=\sum_{\sigma} \rho_{\sigma}, \mathbf{u}=\frac{1}{\rho}\left(\sum_{a=0}^{8} f_{a}^{\sigma} \mathbf{e}_{a}+\frac{1}{2} \sum_{\sigma}\left(\mathbf{F}_{r, \sigma}+\mathbf{F}_{s, \sigma}\right)\right)$. Total system pressure is $p=c_{s}^{2} \rho+\frac{1}{2} c_{s}^{2} \sum_{\sigma \bar{\sigma}} G_{\sigma \bar{\sigma}} \rho_{\sigma} \rho_{\bar{\sigma}}$.

\subsection{Multiscale extension}

In this work, as outlined in Section 2.2, the coupling operator consists of discrete vector quantities, the interfacial forcing terms. Each computational node is assigned a forcing term defined as

$$
\mathbf{F}_{\text {int }}(\mathbf{x}, t)=\frac{1}{6} f_{\text {int }}(\mathbf{x}, t) \mathbf{e}_{a, i n t}(\mathbf{x}, t)
$$

where $f_{\text {int }}(\mathbf{x}, t)$ is the magnitude of the interfacial forcing term and $\mathbf{e}_{a, i n t}(\mathbf{x}, t)$ is a vector that specifies the direction of the forcing term at a given location. Magnitudes of the forcing term may vary or be constant throughout the computational domain. If the dispersed phase is accelerated, the direction vector becomes the same as the bulk flow discrete velocity vector in the flow direction, here $y$ (Eq.3). The interfacial forcing term for the $i^{\text {th }}$ node can be written as,

$$
\mathbf{F}_{\text {int }}^{i}=\frac{1}{6} f_{\text {int }}^{i}\left[\begin{array}{lllllllll}
0 & -1 & 0 & 1 & -1 & -1 & 1 & 1 & 0
\end{array}\right]
$$

Whereas in case of deceleration it is

$$
\mathbf{F}_{\text {int }}^{i}=\frac{1}{6} f_{\text {int }}^{i}\left[\begin{array}{lllllllll}
0 & 1 & 0 & -1 & 1 & 1 & -1 & -1 & 0
\end{array}\right]
$$

All other directions are derived in the same manner. For instance, if the dispersed phase is flowing in the positive $\mathrm{x}$ direction, orthogonal to the main flow, the interfacial forcing term can be expressed as,

$$
\mathbf{F}_{\text {int }}^{i}=\frac{1}{6} f_{\text {int }}^{i}\left[\begin{array}{lllllllll}
1 & 0 & -1 & 0 & 1 & -1 & 1 & 1 & 0
\end{array}\right]
$$


We choose to incorporate the coupling operator into LBM along with the bulk driving force due to flexibility of direction choices and clarity. With the inclusion of the coupling operator Eq.9 becomes,

$$
f_{a}^{\sigma^{\prime \prime}}(\mathbf{x}, t)=f_{a}^{\sigma^{\prime}}+\mathbf{F}_{b u l k}+\mathbf{F}_{\text {int }}(\mathbf{x}, t)=f_{a}^{\sigma^{\prime}}+\frac{1}{6}\left[f_{b u l k} \mathbf{e}_{a, b u l k}+f_{\text {int }}(\mathbf{x}, t) \mathbf{e}_{a, i n t}(\mathbf{x}, t)\right]
$$

Again, this force addition step follows the collision step whereas $f_{a}^{\sigma^{\prime \prime}}(\mathbf{x}, t)$ enters the streaming part of the algorithm. The notation used in Eq.18 highlights the assumption of that the bulk driving force is independent of time and space as opposed to the interfacial forcing term which is allowed to vary with both.

\subsection{Fluid properties and implementation}

We use the fluid properties developed in Huang et al. (2007) with densities and viscosities of both fluids being equal. This scenario is often assumed in the LBM simulations and has been proven to be successful in many applications (Martys and Chen, 1996; Pan et al., 2004; Porter et al., 2009).

The bulk density, $\rho_{b}$, of both fluids is 2.0 and the dissolved density, $\rho_{d}$ is 0.06 . The kinematic viscosity is

$\frac{1}{6}$ and the repulsive interaction strength, $G_{\sigma \bar{\sigma}}$ is 0.9 , which leads to a surface tension of 0.18 . The adsorption potential for fluid-solid interactions, $G_{s, \sigma}$ is 0 or -0.4 for the continuous fluid and 0 or 0.4 for the droplet. For clarity, we refer to the continuous phase as liquid and dispersed phase as droplet, since unit viscosity ratios make the two-phases like immiscible liquids. The interfacial nodes that are affected by the multiscale operator were found based on density criterion, $\left(\rho_{l}-\rho_{d p}<0\right) \&\left(\rho_{d p} \leq \rho_{b}\right) \&\left(\rho_{d p}>\rho_{d}\right)$, where $\rho_{d p}$ and $\rho_{l}$ are droplet and liquid/continuous phase densities respectively. This criterion places the cut-off of the interface halfway between the two phases and ensures that the coupling operator will influence several interfacial nodes.

For simulations and data analysis we use in-house programs developed in MATLAB. The programs are fully parallelized and were run on 11-12 Intel Xeon X5690 3.47 GHz processors. Programs for multicomponent LBM were validated with examples from Sukop and Thorne (2007) and some of the results reported by Huang et al. (2007). Here we present one relevant group of validation results that are part of the fluid-solid interaction parameter determination (Fig. 3, Table 1, Eq.6). A liquid droplet is placed near or on a solid substrate and allowed to equilibrate. The resulting static contact angle will vary with applied $G_{s, \sigma}$. We compared our results with Huang et al. (2007) and consider the agreement satisfactory. Contact angle values were measured computationally following a technique in the aforementioned publication and we explain the variations with resolution differences.

\section{Impact of multiscale extension}

To investigate the impact of the proposed multiscale approach on the Lattice Boltzmann method we have performed a series of simulations with various interfacial forcing terms. The forcing terms used had constant magnitudes or linear magnitude gradient. Directions of the forces were along, opposite or orthogonal to 
Table 1: Static contact angles of a liquid dropletas compared with Huang et al. (2007)

\begin{tabular}{|c|c|c|}
\hline$G_{s, l i q u i d}$ & Huang et al. (2007), & Our model, ${ }^{\circ}$ \\
\hline 0.4 & 158.3 & 162.6 \\
\hline 0.3 & 135.1 & 136.7 \\
\hline 0.2 & 117.0 & 117.8 \\
\hline 0.1 & 103.2 & 103.6 \\
\hline-0.1 & 75.3 & 77.2 \\
\hline-0.2 & 59.5 & 61.7 \\
\hline-0.3 & 40.6 & 41.3 \\
\hline-0.4 & 18.9 & 19.8 \\
\hline
\end{tabular}

Figure 3: Formation of static contact angle of a droplet on a solid substrate.

the bulk driving force as shown in Eq. 9-11 respectively. Walls were made highly droplet repellent with fluid-solid interaction parameters (Eq.6) $G_{s, d}=0.4$ and $G_{s, l}=-0.4$.

The numerical setup consists of a droplet positioned in the middle of the domain (Fig. 4). The side boundaries were reflecting walls with no-slip, halfway bounce-back boundary condition and the top and bottom boundaries were periodic. The system was driven with a body force that acted on both fluids, and due to the applied coupling operators, the droplet could accelerate, decelerate or change direction where suitable. The results are presented in Figures 5-7.

Results are shown for two magnitudes of operators $\left(f_{\text {int }}\right)$ relative to the bulk force magnitude $\left(f_{\text {bulk }}\right)$. Fig. 5 shows the results of applying coupling operators with direction opposite to the bulk driving force. The

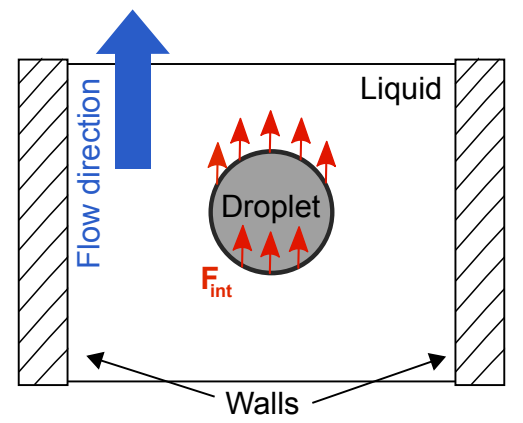

Figure 4: Numerical setup for investigation of the impact of proposed numerical approach on LBM. 

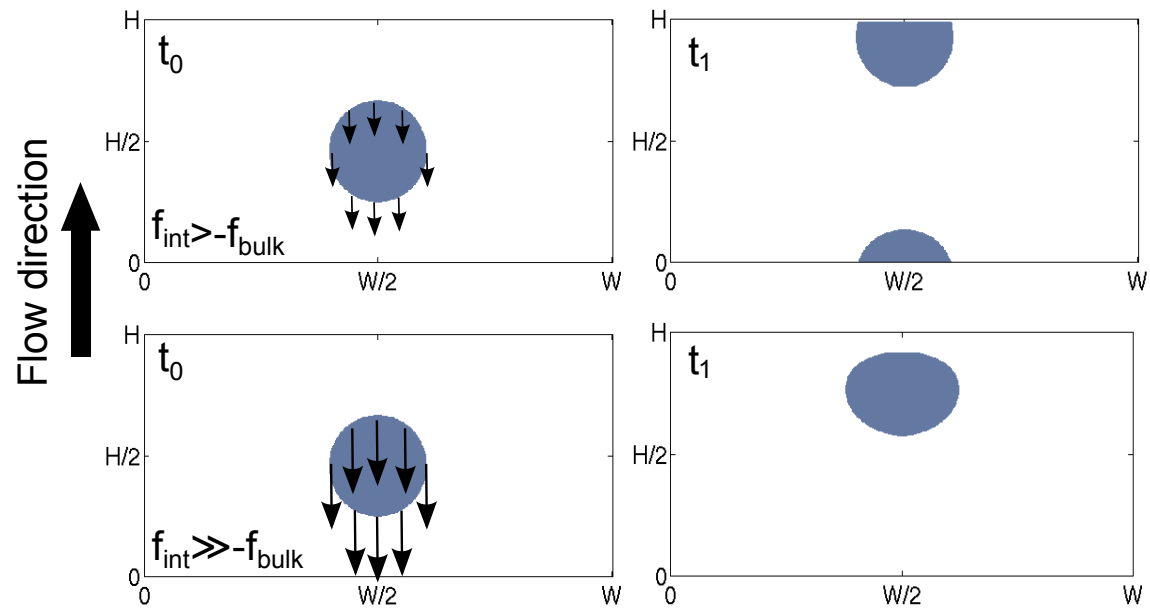

Figure 5: Results of applying coupling operators, $f_{\text {int }}$ with direction opposite to the bulk driving force, $f_{\text {bulk }}$.
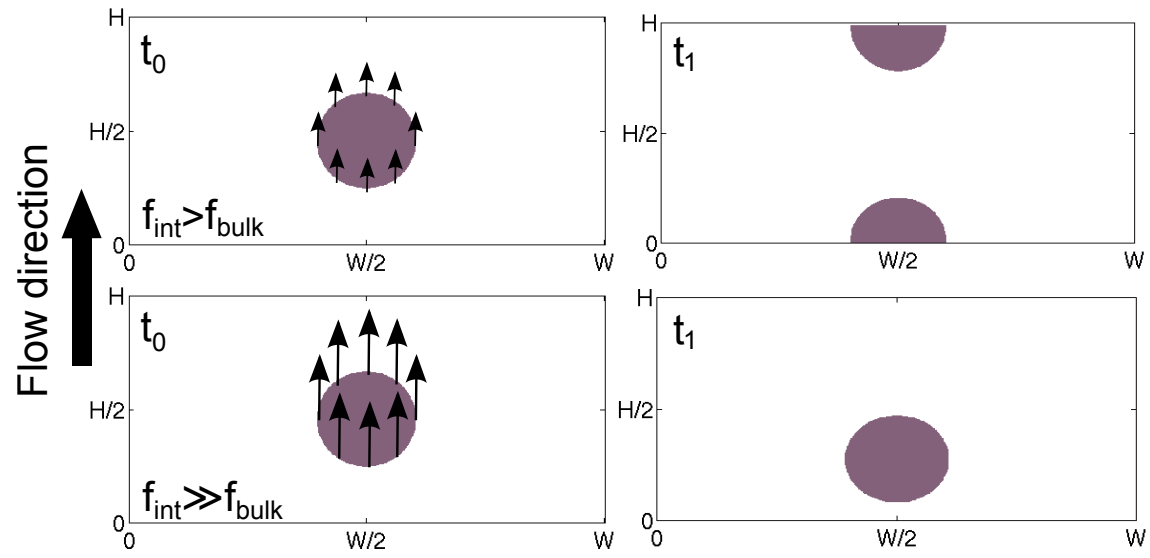

Figure 6: Results of applying coupling operators, $f_{\text {int }}$ with direction parallel to the bulk driving force, $f_{\text {bulk }}$.

droplet affected by the lower magnitude operator re-enters the domain while the droplet under the action of higher magnitude operator deforms and starts flowing in the direction opposite to the flow. Fig. 6 represents the case where the coupling operator has the same direction as the body force. Both droplets accelerate proportionally to the magnitudes applied. Droplet affected by the higher magnitude operator is closer to the domain middle at the time when the less affected droplet is still re-entering the domain. Fig. 7 shows the results of applying coupling operators acting orthogonal to the main flow direction. The larger magnitude operator causes significant deformation of the droplet. The droplet does not come in direct contact with the solid walls due to high repulsive interactions.

Material balance for all coupling operators used closes within less than $0.07 \%$ and there were no instabilities or numerical artifacts detected. The condition used for tracking interfacial nodes tends to include interior portions of the bubble instead of only target interfacial nodes, and the inclusion of interior nodes is caused by slight mass loss due the application of the coupling operator. This phenomenon decreases 
the operator robustness as the thickness of the interface under operator action varies with less control. To negate this, the interface condition can be adaptive to include only fixed number of interfacial nodes to avoid excessive and unpredictable effects of the applied coupling operator. One example would be to use an extended interface locating criterion, namely in addition to the criterion defined in Section 3.3, $F 1=\left(\rho_{l}-\rho_{d p}<0\right) \&\left(\rho_{d p} \leq \rho_{b}\right) \&\left(\rho_{d p}>\rho_{d}\right)$, a second criterion could be used, $F 2=\rho_{d p}>\rho_{0} . \rho_{0}$ is a density threshold that by value is closer to bulk droplet density, for instance if the bulk density is $2.0, \rho_{0}$ can be 1.95. With these two criteria, interfacial nodes influenced by the coupling operator are nodes for which $F 1-F 2>0$. This extended condition is both more accurate and stable, but also computationally more intensive. The initial number of interfacial nodes can be recorded, and if it increases significantly during the simulation, the number of interfacial nodes can be changed by varying the $\rho_{0}$ threshold. This condition can also be used as it is, without the adaptiveness.

The magnitudes of the forcing term have a broad enough span and droplets do respond to coupling operators proportionally to forcing term magnitudes and consistently with the force directions.

\section{Multiscale modeling of domains with posts}

In this section we present simulations results of a droplet flowing through arrays of solid posts and subsequent applications of our multiscale modeling approach on these model systems. We use two post configurations (Figure 8) with different number and dimensions of posts. Top and bottom boundaries are periodic and sides are no-slip walls. $G_{s, \sigma}$ is zero for both components ensuring neutral affinity of both fluids towards the solid features. The coarse scale domain is empty with walls only. Sizes of fine and coarse domains
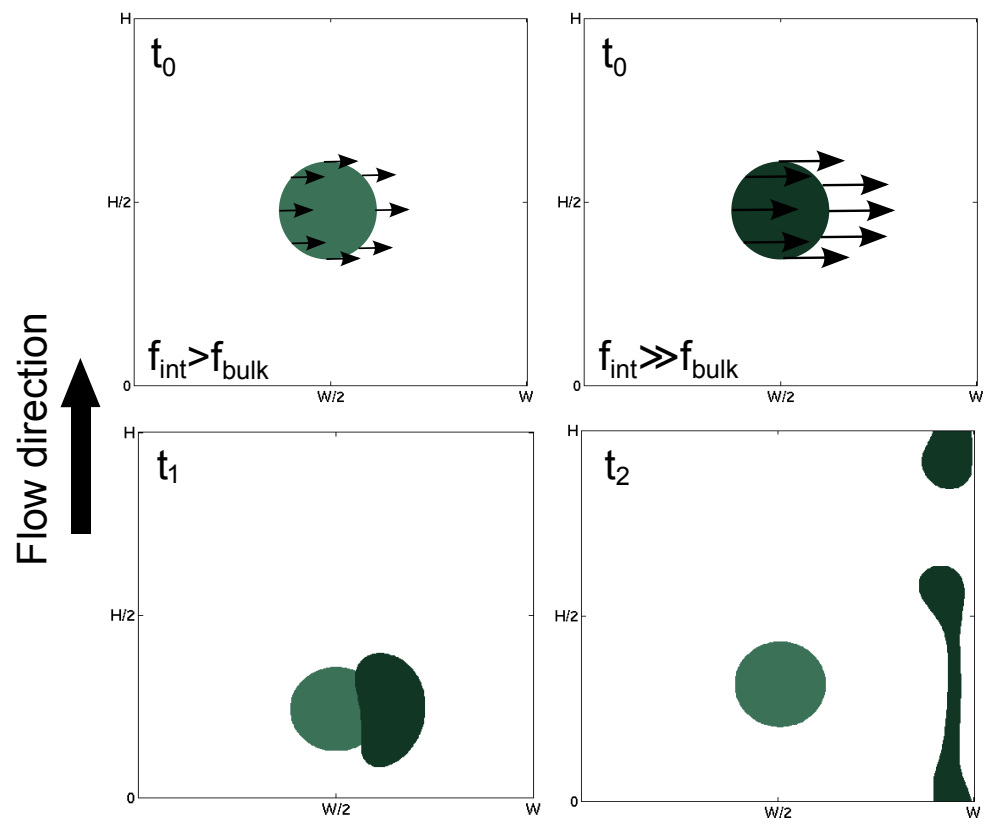

Figure 7: Results of applying coupling operators, $f_{\text {int }}$ with direction orthogonal to the bulk driving force, $f_{b u l k}$. 
were $600 \times 600$ lattice units (lu). Multiscale modeling of a coarse scale with coarser grid is discussed in Section 6.2. The droplet has a radius of $R_{d}=62.25$ lu for all simulations in this section and it is initially placed in the center of the domain, upstream from the post array.

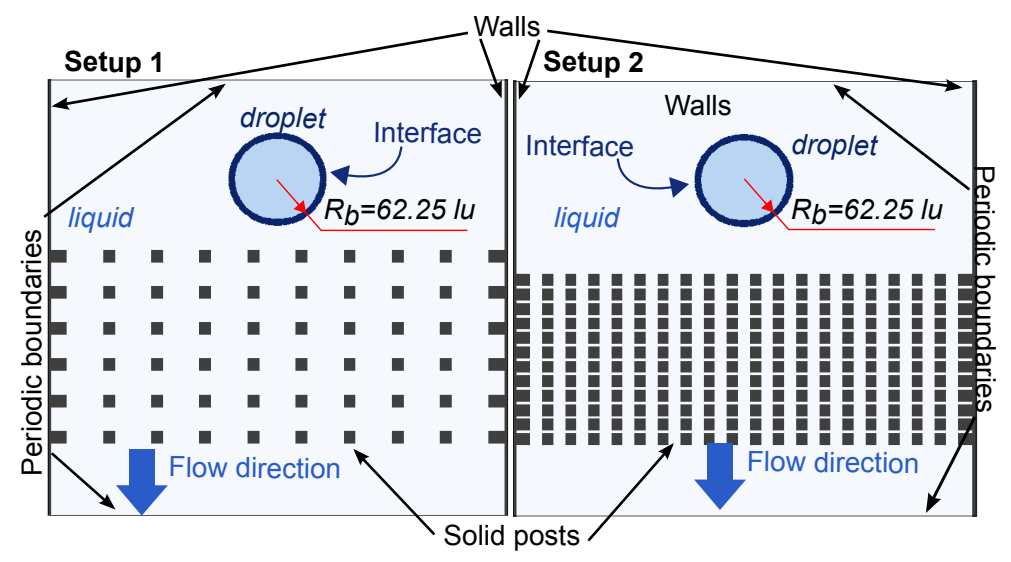

Figure 8: Two post configurations with setup description and droplet in its initial position.

\subsection{Droplet retention times}

Retention times are computed based on the position of nodes that contribute to the interface and are summarized in Table 2. We define several time intervals:

- Pre-entrance period, $\mathrm{T}_{1}$

The time from when the droplet is released until the first interface node enters the post region.

- Entrance, $\mathrm{T}_{2}$

The time from the end of the pre-entrance period until last interface node enters the post region.

- Bulk, $T_{3}$

The period from the end of entrance period until the first node of the interface exits the posts region.

- Exit, $\mathrm{T}_{4}$

From the end of the bulk period until the last interface node exits the post region.

- Total time in contact with posts, $\tau_{d}$

Summation of the entrance, bulk, and exit periods.

Table 2 also contains corresponding values computed for the equivalent domain without posts.

In all four simulation setups (S1, S2, ES1, and ES2) the Reynolds number of the flow at the inlet and outlet was 140, but despite this the droplet retention time in sparse post array (Setup 1) was more than twice that of the dense array (Setup 2). The major difference appears in the bulk and the exit periods. Reason for this behavior is the neutral affinity of the fluids towards the solid posts. While there is large energy associated with the liquid-droplet interface, the surface energies of the liquid-post and the droplet-post interfaces are 
Table 2: Droplet retention times

\begin{tabular}{llllll}
\hline Setup & $\mathrm{T}_{1}, \mathrm{ts}$ & $\mathrm{T}_{2}, \mathrm{ts}$ & $\mathrm{T}_{3}, \mathrm{ts}$ & $\mathrm{T}_{4}, \mathrm{ts}$ & $\tau_{d}, \mathrm{ts}$ \\
\hline Setup 1 (S1) & 4,400 & 9,500 & 23,600 & 27,600 & 56,300 \\
Setup 2 (S2) & 7,600 & 8,000 & 3,500 & 9,200 & 20,700 \\
Empty based on S1 (ES1) & 6,300 & 5,800 & 7,900 & 9,000 & 22,700 \\
Empty based on S2 (ES2) & 9,200 & 5,800 & 5,900 & 8,100 & 19,800 \\
\hline
\end{tabular}

identical. However, although a post has no affinity for a droplet over the liquid the posts are able to pin the droplet as a post intersecting the droplet boundary replaces some of the droplet-liquid interface with a boundary that must exist regardless of the droplet configuration. The result is a net reduction in surface, and thus, for any droplet-liquid surface energy larger than zero there will be a pinning effect (regardless of the droplet-liquid surface energy).

A droplet moving into an array of posts provides two mechanisms for changing the total interfacial energy of the system. Posts that are subsumed into the droplet take up space causing the outer droplet boundary to expand and increasing the energy of the system. However, this increase in energy can be offset by the replacement of high energy droplet-liquid interface with an effectively zero energy droplet-post boundary. The posts are identical in their interaction with the droplet and the work needed to pass a post is equivalent to the balance of pinning and depinning forces summed with frictional losses. In a sparse array of posts the droplet must overcome the force to break free of each post as it moves forward. However, in the dense array of posts there are multiple low energy configurations of the droplet boundary between the posts, and it is not necessary for the droplet to completely break free of each post, before it can adhere to the next. Thus in the dense array of posts the work that must be provided to move the droplet forward can be considerably less than in the case of the sparse array.

In Setup 1 where the posts are sparsely distributed the droplet needs considerably more time to overcome pinning forces and move on to the next posts. The rear of the droplet regularly develops highly flow resistant concave curvature. In Setup 2 the dense array of posts provides no such opportunity during bulk and exit periods. Droplet flow through both configurations is shown in Figure 9. Additionally, in the exit period in Setup 1 formation of concave rear is always accompanied by highly negative pressure drops which by convention used here indicates even higher droplet resistance to flow (Fig. 10). In Setup 2, small negative capillary pressure drops occur only during the entrance period. This detailed analysis of the flow of the droplet through the post array is technologically important as it demonstrates that the shape and size of posts can be designed to provide size selective forces on secondary phases that can be used to provide novel means for filtering, segregating, and corralling these secondary phases from within the carrier flow. 


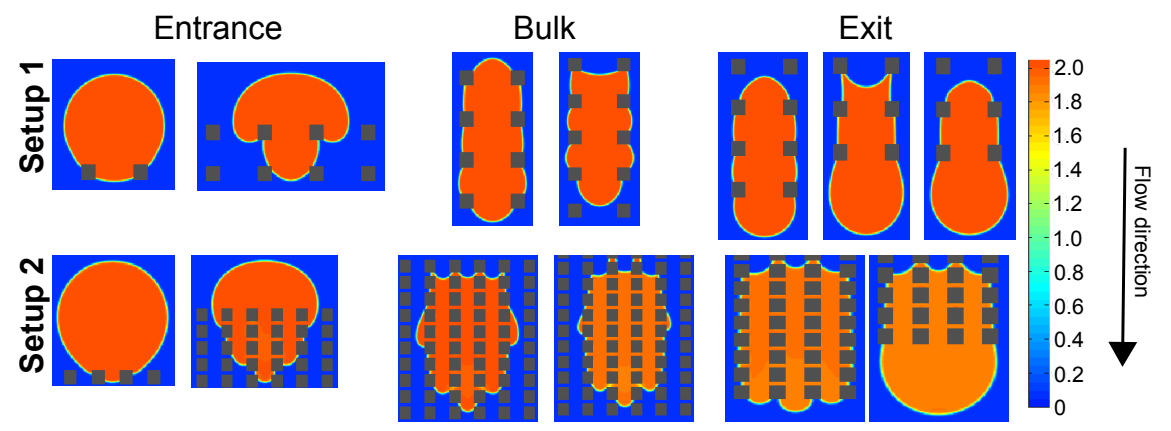

Figure 9: Droplet flowing through two post configurations.
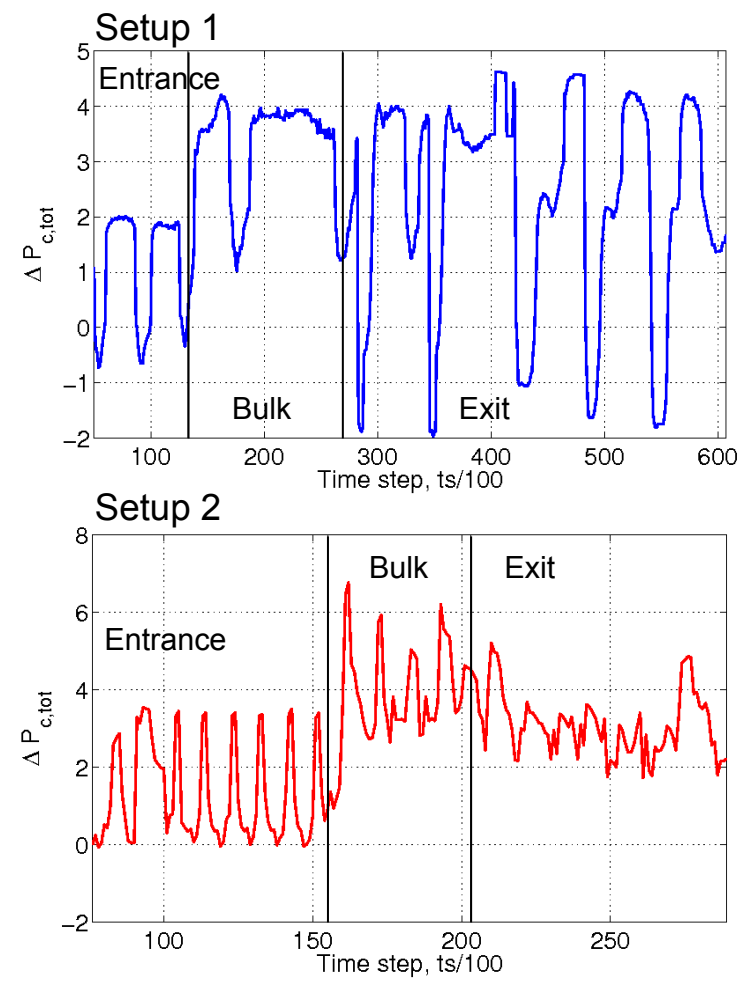

Figure 10: Total normalized capillary pressure drops across the droplet in time for both setups. Total pressure drops form distinguishable patterns during three droplet flow periods, entrance, bulk and exit. Highly negative pressure drops indicate additional flow resistance.

\subsection{Scaling of LBM results to physical quantities}

The simulations presented here have all been performed within a system of LBM units - chosen somewhat arbitrarily rather than representing a particular experimental setup. Thus here we show that the LBM parameters chosen do map to physically meaningful system.

The physical spatial dimension per lattice unit is $d_{h}=\frac{d_{p}}{d_{L B M}}, \frac{\mu m}{l u}$ and temporal scaling is, $t_{h}=$ $\rho_{l} \nu_{L B M} \frac{d_{h}^{2}}{\mu_{l}}, \frac{s}{t s}$ (Porter et al., 2009). Here $d_{p}$ is a dimension in physical units, for example, length of the device, and $d_{L B M}$ is the same dimension in lu. $\nu_{L B M}=\frac{1}{6} \quad \frac{\mathrm{lu}^{2}}{\mathrm{ts}}$ is the kinematic viscosity (Section 3.1). $\rho_{l}$ and $\mu_{l}$ are density and viscosity of the continuous phase in physical units. 
Assuming properties of water at room temperature, $\rho_{l}=1000 \mathrm{~kg} / \mathrm{m}^{3}, \quad \mu_{l}=1.0 \times 10^{-3} \mathrm{~Pa} \cdot \mathrm{s}$ and taking $d_{p}$ to be width of a post in Setup 1 measuring $500 \mu m$ we can compute several physical quantities. Post width in Setup 2 becomes $375 \mu \mathrm{m}$, size of both domains is $2.5 \times 2.5 \mathrm{~cm}$ and initial droplet diameter is $0.5 \mathrm{~cm}$. Droplet retention time, $\tau_{d}$ in Setup 1 is $16.3 \mathrm{~s}$ and in Setup $26.0 \mathrm{~s}$. Dividing the length of the post region with the retention time yields approximate droplet velocity: $0.07 \mathrm{~cm} / \mathrm{s}$ in Setup 1 and $0.16 \mathrm{~cm} / \mathrm{s}$ in Setup 2. The continuous liquid flow velocity as computed from the Reynolds number is $0.28 \mathrm{~cm} / \mathrm{s}$. We consider the obtained values plausible.

\subsection{Multiscale modeling}

To demonstrate the proposed multiscale modeling method we have found interfacial forcing terms values for the two setups. Interfacial forcing terms were found via iterative - self-consistent process such to yield the same total bubble retention times in equivalent geometry. Interfacial forcing terms were non-zero in the region that would otherwise correspond to post array region and a single forcing term magnitude was found for each setup. For Setup 1 the magnitude value was $1.27 \times 10^{-4}$ which corresponds to $209 \times f_{\text {bulk }}$ in the equivalent domain. For Setup 2 the magnitude was $1.58 \times 10^{-5}$ which corresponds to $26 \times f_{\text {bulk }} \cdot f_{\text {bulk }}$ and Re were the same for ES1 and ES2.

Additionally, for Setup 2 we developed a forcing term distribution to match the droplet flow periods presented in Table 2. The distribution consisted of constant interfacial forcing term magnitude values within specified sections of the entire domain. The obtained forcing term magnitudes were large compared to the driving force magnitude and caused significant droplet deformation in the bulk and exit periods. Furthermore, during the simulation, interface condition started covering interior nodes of the droplet which may in general inversely influence the results and robustness. Thus it does not seem that constant magnitude distribution is suitable for at least this setup. Potentially more superior distributions could include linear, exponential or other, smoother functional dependencies of the magnitudes with space.

\section{Multiscale modeling results}

In this section we present a study of the robustness of the proposed approach. We use the interfacial forcing term magnitude for Setup 2 reported above on different droplet sizes with fixed Re and different Re for the same droplet size. Additionally we perform multiscale modeling with an upper domain with coarser computational grid. Finally we demonstrate successful construction of local subdomains for piecewise modeling of the system from Section 5, also for Setup 2.

\subsection{Robustness of the multiscale method}

The robustness of the method was investigated by applying the coupling operator reported in Section 5.3 in a geometry corresponding to Setup 2. Two groups of cases were investigated with smaller Re and smaller droplet diameters. Smaller Re included applying originally developed coupling operator on a droplet 
of the same dimensions as in Section 5 in a system with lower velocities. Numerical experiments with smaller droplet sizes involved applying a scaled coupling operator on a droplet with the same Re as in the Section 5 runs.

\subsubsection{Lower Re simulations}

The Reynolds numbers used were 8 and 70 whereas the original Re was 140 . Our choice of Re was such that in one case the droplet would not manage to enter the post array whereas in the other it would flow through the post region with a visibly lower velocity. The forcing terms opposed the bulk flow direction with magnitude of $f_{i n t}=1.58 \times 10^{-5}$. For $R e=8.0$ the droplet did not enter the post region. Application of the coupling operator reproduces this behavior exactly in the coarse scale level. In case of $R e=70.0$, the retention time of the droplet in the fully resolved domain with posts was 56,100 ts and in the equivalent, empty, domain upon application of the upscaling operator it was 50,200 ts. This corresponds to a $10.52 \%$ relative error. For a comparison, the droplet retention in an empty domain with no coupling operator applied was 36,200 ts which is considerably less than in the actual domain with posts.

\subsubsection{Smaller droplet dimensions simulations}

Two smaller droplets were simulated with radiuses of $27.5 \mathrm{lu}$ and $56.25 \mathrm{lu}$ (compared to the original $62.25 \mathrm{lu}$ ). These simulations with smaller radius yielded excellent agreement when applying the upscaling operator, a radius close to the original value was also tested to verify its performance. Re was fixed for all three setups to about 140.0. Results are displayed in Table 3 along with tier comparison with values for the original radius, $R_{0} . \tau_{d, p}$, ts represents total time in contact with posts on the fine scale as defined in Section 5.1. $\tau_{d, e}, t s$ is the total time in contact with posts on the coarse scale without coupling operator applied to the droplet interface, $f_{i n t}=0 . \tau_{d, m}, t s$ is the time in contact with post on the coarse scale with coupling operator applied on droplet interface. As the size of a droplet is changed we need to include a proportionality factor to scale the original forcing term magnitude $f_{\text {int }, 0}$ reported in Section 5.3. The factor assumes linear change and was defined as $\left(\frac{R_{0}}{R_{d}}\right)$ where $R_{d}$ is the new radius, either 27.5 or $56.25 l u$. The adjusted forcing term magnitude becomes $f_{\text {int }}=\left(\frac{R_{0}}{R_{d}}\right) \times f_{\text {int }, 0}$.

Table 3: Results of lower radius simulations with relative differences.

\begin{tabular}{lllll}
\hline Droplet radius, $l u$ & $\tau_{d, p}$, ts & $\tau_{d, e}, t s$ & $\tau_{d, m}, t s$ & $\frac{\tau_{d, p}-\tau_{d, m}}{\tau_{d, p}} \times 100, \%$ \\
\hline 27.5 & 18,950 & 14,500 & 19,000 & -0.26 \\
56.25 & 21,700 & 22,600 & 14,400 & -4.1 \\
62.25 & 20,700 & 20,700 & - & 0 \\
\hline
\end{tabular}

\subsection{Multiscale modeling with a coarse upper level}

In addition to multiscale modeling with the upper level having the same grid size as the lower, we have performed simulations with a coarse upper level. This is the ultimate goal of our two level approach. The 
upper level was an empty domain with walls on the sides and its size was $300 \times 300 l u$. Fine scale was Setup 2 described in Section 5.1.

We developed the coupling operator based on the simulation with $R e=140$ and $R_{d}=62.25 \mathrm{lu}$ which we approximated with $R_{d}=32 \mathrm{lu}$ on the coarse scale. The coupling operator was found to be $1.08 \times 10^{-4}$ or $27 \times f_{\text {bulk }}$, which is a ratio remarkably close to the one obtained for S2 without the coarser grid (Section 5.3.).

In LBM spatial and temporal resolution are coupled, hence to develop the operator and perform a robustness study droplet retention times on the fine scale have to be converted to physical units from which they are re-converted into LBM time steps on the coarse scale. This is done using the spatial and temporal scaling relation introduced in Section 5.2. Again assuming that the continuous liquid is water, the time step conversion factor for S2 becomes $2.8935 \times 10^{-4} \frac{s}{t s}$ and the one for new coarse scale is $1.1574 \times 10^{-3} \frac{s}{t s}$. Notice that due to square dependency of time step on the grid size, the coarse scale time step is four times larger. This signifies that running the simulation until some fixed physical time on the coarse scale will not only take less computational effort due to coarser spatial grid but also due to increased LBM time step.

Robustness study was performed for three cases as in Section 6.1. In the first case, Re was about 70 and the droplet radius was the same as in the original setup, $R_{d}=62.25 \mathrm{lu}$ on the fine scale and $R_{d}=32 \mathrm{lu}$ on the coarse scale. Compared was the total time in contact with posts. The relative error between fine and coarse scales measured 32.1\%. A second case included the same droplet radius with even lower Re of 8.0. On the fine scale the droplet did not cross the post array, on the coarse scale it did, but only after a very long time period. Finally, in the third study the Re measured 140.0 but the droplet radius was $27.5 \mathrm{lu}$ on the fine scale and $14.0 \mathrm{lu}$ on the coarse scale. The relative error between those two cases was $7.1 \%$. In all three of the cases in this robustness study increasing the coarseness of the lattice resulted in higher errors compared to the simulations with coarse scale with the same grid as the fine scale. Nevertheless, the size of the errors is still be sufficiently low for many applications.

\subsection{Construction of local subdomains}

The concept of piecewise modeling is schematically displayed in Figure 11. Piecewise modeling consists of modeling only part of the original domain as outlined in Section 2.3. For a given architecture one can select it's characteristic regions, most relevant to the flow of dispersed phase, model them separately, and then extrapolate the flow information to cover the entire device. While the idea of piecewise modeling is not novel, applying it to fully resolved flow of bubbles or droplets is yet to be explored. Here we present results of the first piecewise modeling step, namely constructing local subdomains for Setup 2 geometry for $R_{d}=62.25 \mathrm{lu}$ and Reynolds number about 140. Besides rules for creating the subdomains, we evaluate the performance of subdomains compared to original system.

Based on numerical experiments we have established several rules for successful generation of local subdomains thus far: 


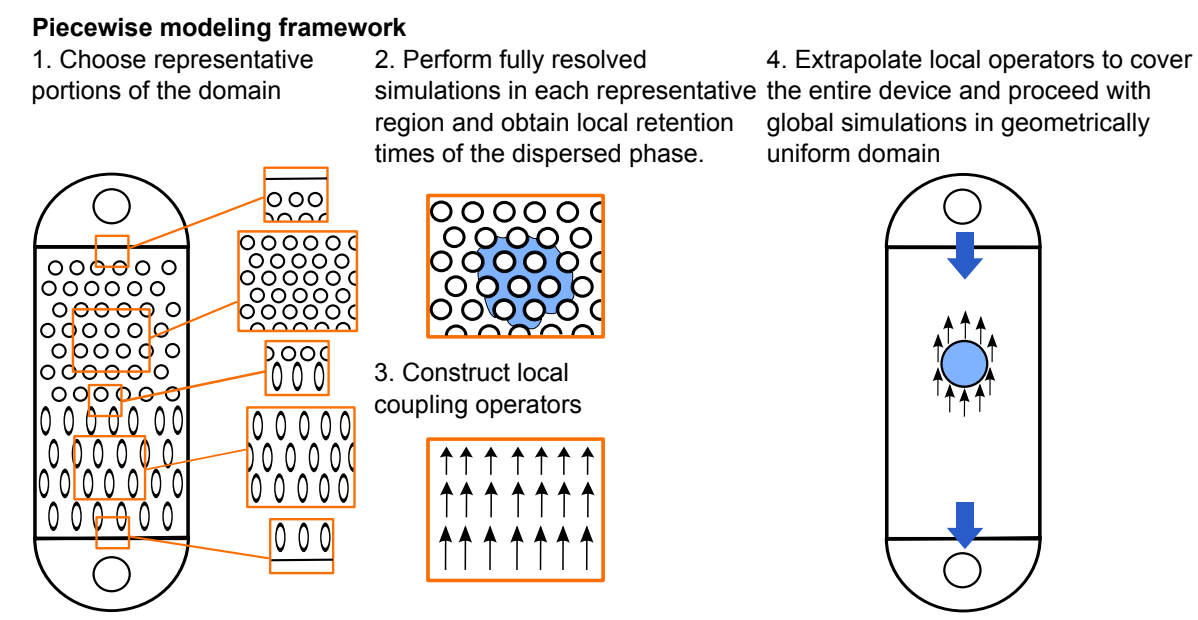

Figure 11: Piecewise modeling procedure.

1. The width of the local subdomain and volumetric flow rate have to comply with the global scale width. These two conditions make local flow of continuous phase equivalent to the system level.

2. The droplet has to be initially placed at the same distance relative to the post array.

This way the droplet enters the post region with momentum close to one in the whole domain simulations.

3. The post region in local subdomain has to fully contain the bubble and let it experience its steady state flow dynamics in the post array.

In the presented simulations the inlet, bulk and outlet from the post region were simulated within the same run with some space for the bulk flow. The bulk flow is compared as droplet velocity computed from times and distances travelled by droplet front. In the original size setup the bulk flow was $0.0174 \mathrm{lu} / \mathrm{ts}$.

The new domain was $600 \times 442 \mathrm{lu}$ instead of the original $600 \times 600 \mathrm{lu}$, with the post array spanning from $y=235 l u$ to $y=425 l u$ which was $40 l u$ shorter than the fine scale domain. The results were $8,100 \mathrm{ts},-1.25 \%$ error for the inlet period and exact timing for the exit, and the bulk velocity was $0.0186 \mathrm{lu} / \mathrm{ts}$ with relative error of $6.90 \%$.

Since the above listed rules were derived based on simulations with $R_{b}=62.25$ lu to further test their applicability we have constructed a local subdomain for the $R_{b}=27.5 l u$ case. The local subdomain was $600 \times 286 l u$ and the posts spanned from $y=159 l u$ to $y=269 l u$. The inlet and exit times were the same as in the original simulation while droplet bulk velocity was $0.0164 \mathrm{lu} / \mathrm{ts}$, differing by $3.8 \%$.

In the above we have shown that the proposed piecewise modeling provides potentially sufficient modeling accuracy while considerably lowering computational effort. Moreover, it can be used as a stand-alone tool for two-phase flow characterization or as a fine, lower level in multiscale modeling. 


\section{Conclusions and outlook}

We presented a novel, multiscale modeling approach applicable for the two-phase flow in microscale devices with microfeatures applied within SC LBM. This approach was implemented within Shan and Chen Lattice Boltzmann model, and it was demonstrated by simulation of retention times of a dispersed phase flowing through microscale post array.

The demonstration of the proposed multiscale modeling approach involves a coupling operator in the form of discrete vector fields applied at the interface of the dispersed phase. Coupling operator was built into SC LBM as a source term, and defined as non-zero only at the interfacial nodes. Inclusion of the coupling operator did not cause instabilities and its impact on conservation properties was satisfactory. Tests with multiple coupling operators of various magnitudes and directions verified that a droplet behavior is sensitive to the directions and magnitudes of the applied operator.

Multiscale modeling was applied to two model systems with rectangular posts of different spacing and dimensions. Simulation results were physically plausible which was confirmed through scaling of selected quantities from LB to physical units. Coupling operators were obtained for both setups. Operators provided the same retention times of the droplet in the geometrically uniform domain as in the post region of fully resolved domain including the posts. Developed coupling operators were also tested for their robustness yielding good accuracy in all investigated cases.

We also demonstrated the concept of piecewise modeling of microarchitectures with posts. Rules for piecewise modeling were developed and supported with sufficiently accurate numerical results.

Proposed approach can be used for characterization of microscale-based structures and for more efficient optimization of the design and operating parameters of processes performed in microscale-based structures. A broader applications of the proposed multiscale modeling, attained through further development, could facilitate inverse construction of microarchitectures based on simulations on the upper level and corresponding coupling operators. In other words, geometric characteristics of post arrays could be determined from the coupling operator values and distributions. This would enable efficient design optimization, as most of the computational effort would shift to the coarser (upper) scale. Manipulation of the coupling operator would induce specific behavior of the dispersed phase, which would then be translated into specific design of microscale features in the fully resolved domain.

\section{Acknowledgements}

This work was supported in part by the National Institute of Biomedical Imaging and Bioengineering (grant no. R01EB011567). The content is solely the responsibility of the authors and does not necessarily represent the official views of the National Institute of Biomedical Imaging and Bioengineering, or the National Institutes of Health. Additionally, we acknowledge the funding obtained from U.S. Department of Energy through Hydrogen Storage Engineering Center of Excellence, grant no: DE-FC36-08GO19005. We, also, gratefully acknowledge the funding obtained from: ONAMI (Oregon Nanoscience and Microtechnologies In- 
stitute) and Oregon State University College of Engineering. Lastly, we acknowledge Dr. Liney Arnadottir and Jennifer Fogarty for proofreading of this manuscript. 


\section{References}

Buick, J., Greated, C., 2000. Gravity in a lattice Boltzmann model. Physical Review E 61 (5), 5307.

Chung, S. K., Zhao, Y., Cho, S. K., 2008. On-chip creation and elimination of microbubbles for a micro-object manipulator. Journal of Micromechanics and Microengineering 18 (9), 095009.

De Loos, S., Van Der Schaaf, J., Tiggelaar, R., Nijhuis, T., De Croon, M., Schouten, J., 2010. Gas-liquid dynamics at low reynolds numbers in pillared rectangular micro channels. Microfluidics and nanofluidics 9 (1), 131-144.

Doku, G. N., Verboom, W., Reinhoudt, D. N., Van Den Berg, A., 2005. On-microchip multiphase chemistry a review of microreactor design principles and reagent contacting modes. Tetrahedron 61 (11), 2733-2742.

Gravesen, P., Branebjerg, J., Jensen, O. S., 1993. Microfluidics-a review. Journal of Micromechanics and Microengineering 3 (4), 168.

Günther, A., Jensen, K. F., 2006. Multiphase microfluidics: from flow characteristics to chemical and materials synthesis. Lab on a Chip 6 (12), 1487-1503.

Hartman, R. L., Jensen, K. F., 2009. Microchemical systems for continuous-flow synthesis. Lab on a Chip 9 (17), 2495-2507.

Hessel, V., Angeli, P., Gavriilidis, A., Löwe, H., 2005. Gas-liquid and gas-liquid-solid microstructured reactors: contacting principles and applications. Industrial \& engineering chemistry research 44 (25), 97509769.

Huang, H., Sukop, M., Lu, X., 2015. Multiphase lattice Boltzmann methods: Theory and application. John Wiley \& Sons.

Huang, H., Thorne, D., Schaap, M. G., Sukop, M. C., 2007. Proposed approximation for contact angles in Shan-and-Chen-type multicomponent multiphase lattice Boltzmann models. PHYSICAL REVIEWSERIES E- 76 (6), 066701.

Huh, D., Tung, Y.-C., Wei, H.-H., Grotberg, J. B., Skerlos, S. J., Kurabayashi, K., Takayama, S., 2002. Use of air-liquid two-phase flow in hydrophobic microfluidic channels for disposable flow cytometers. Biomedical Microdevices 4 (2), 141-149.

Jensen, K. F., 2001. Microreaction engineering - is small better? Chemical Engineering Science 56 (2), 293-303.

Jensen, M. J., Goranović, G., Bruus, H., 2004. The clogging pressure of bubbles in hydrophilic microchannel contractions. Journal of Micromechanics and Microengineering 14 (7), 876. 
Kiwi-Minsker, L., Renken, A., 2005. Microstructured reactors for catalytic reactions. Catalysis Today 110 (1), $2-14$.

Kohnle, J., Waibel, G., Cernosa, R., Storz, M., Ernst, H., Sandmaier, H., Strobelt, T., Zengerle, R., 2002. A unique solution for preventing clogging of flow channels by gas bubbles. In: Micro Electro Mechanical Systems, 2002. The Fifteenth IEEE International Conference on. IEEE, pp. 77-80.

Kolb, G., Hessel, V., 2004. Micro-structured reactors for gas phase reactions. Chemical Engineering Journal $98(1), 1-38$.

Krishnamurthy, S., Peles, Y., 2007. Gas-liquid two-phase flow across a bank of micropillars. Physics of Fluids (1994-present) 19 (4), 043302.

Krishnamurthy, S., Peles, Y., 2008. Flow boiling of water in a circular staggered micro-pin fin heat sink. International Journal of Heat and Mass Transfer 51 (5), 1349-1364.

Lam, K. F., Sorensen, E., Gavriilidis, A., 2013. Review on gas-liquid separations in microchannel devices. Chemical Engineering Research and Design 91 (10), 1941-1953.

Litterst, C., Kohnle, J., Ernst, H., Messner, S., Sandmaier, H., Zengerle, R., Koltay, P., 2004. Improved gas bubble mobility in chic-type flow channels. ACTUATOR. Messe Bremen, Germany, 541-544.

Martys, N. S., Chen, H., 1996. Simulation of multicomponent fluids in complex three-dimensional geometries by the lattice Boltzmann method. Physical Review E 53 (1), 743.

Pan, C., Hilpert, M., Miller, C., 2004. Lattice-Boltzmann simulation of two-phase flow in porous media. Water Resources Research 40 (1).

Porter, M. L., Schaap, M. G., Wildenschild, D., 2009. Lattice-Boltzmann simulations of the capillary pressure-saturation-interfacial area relationship for porous media. Advances in Water Resources 32 (11), $1632-1640$.

Shan, X., Chen, H., 1993. Lattice Boltzmann model for simulating flows with multiple phases and components. Physical Review E 47 (3), 1815.

Sparks, D., Cruz, V., Najafi, N., 2007. The resonant behavior of silicon tubes under two-phase microfluidic conditions with both microbeads and gas bubbles. Sensors and Actuators A: Physical 135 (2), 827-832.

Sukop, M. C., Thorne, D. T., 2007. Lattice Boltzmann modeling: an introduction for geoscientists and engineers. Springer.

Wang, W., Liu, Z., Jin, Y., Cheng, Y., 2011. Lbm simulation of droplet formation in micro-channels. Chemical Engineering Journal 173 (3), 828-836. 
Wiesegger, L., Knauss, R., Guntschnig, G., Brandner, J., Marr, R., 2013. Vapor-liquid phase separation in micro-/ministructured devices. Chemical Engineering Science 93, 32-46.

Yu, Z., Hemminger, O., Fan, L.-S., 2007. Experiment and lattice Boltzmann simulation of two-phase gasliquid flows in microchannels. Chemical Engineering Science 62 (24), 7172-7183.

Zhao, Y., Chen, G., Yuan, Q., 2006. Liquid-liquid two-phase flow patterns in a rectangular microchannel. AIChE journal 52 (12), 4052-4060. 